\title{
组织驻留记忆T细胞与肿瘤研究进展
}

\author{
吴琼丽，康双朋，吴长有* \\ 中山大学中山医学院免疫学研究所, 广州 510080 \\ *联系人, E-mail: changyou_wu@yahoo.com \\ 收稿日期：2020-03-19; 接受日期：2020-04-06; 网络版发表日期：2020-07-23 \\ 国家自然科学基金(批准号: 81971556)资助
}

摘要 免疫应答之后, 大多数效应 $\mathrm{T}$ 细胞凋亡, 只有少数细胞形成记忆 $\mathrm{T}$ 细胞. 根据其分布、功能和转录因子的不 同，可将记忆 $\mathrm{T}$ 细胞分为效应记忆 $\mathrm{T}$ 细胞(effector memory $\mathrm{T}$ cells, $\mathrm{T}_{\mathrm{EM}}$ )、中央记忆 $\mathrm{T}$ 细胞(central memory $\mathrm{T}$ cells, $\mathrm{T}_{\mathrm{CM}}$ ) 和组织驻留记忆 $\mathrm{T}$ 细胞(tissue resident memory $\mathrm{T}$ cells, $\mathrm{T}_{\mathrm{RM}}$ ). $\mathrm{T}_{\mathrm{RM}}$ 细胞是非循环的淋巴细胞, 分布于非淋巴组织 中, 包括皮肤和黏膜组织、泌尿生殖系统、脑组织、呼吸系统、肺和肝脏等组织中. 当病原微生物入侵后, 能够 直接和快速产生防御反应. 此外, $\mathrm{T}_{\mathrm{RM}}$ 细胞在肿瘤的免疫、变态反应性疾病和自身免疫性疾病的发生中也发挥十 分重要的功能. $\mathrm{T}_{\mathrm{RM}}$ 细胞包括 $\mathrm{CD} 4^{+}$和 $\mathrm{CD} 8^{+} \mathrm{T}$ 细胞, 表达 CD69和/或 CD103, 在免疫应答过程中, 产生细胞因子和/或 杀伤功能, 连接天然免疫和过继免疫, 启动全身性免疫应答. 此外, 研究表明 $\mathrm{T}_{\mathrm{RM}}$ 细胞的功能受组织中调节性 $\mathrm{T}$ (regulatory $\mathrm{T}, \mathrm{Treg}$ ) 细胞的调控. 为此, 本文对 $\mathrm{T}_{\mathrm{RM}}$ 细胞的分化及在肿瘤方面的作用进行综述.

关键词 $\mathrm{T}_{\mathrm{RM}}$, 肿瘤免疫, 疫苗

经典的分类方法根据记忆 $\mathrm{T}$ 细胞的表型、循环路 径和效应功能不同将记忆 $\mathrm{T}$ 细胞分为两群，即中央记 忆 $\mathrm{T}$ 细胞(central memory $\mathrm{T}$ cells, $\mathrm{T}_{\mathrm{CM}}$ ) 和效应记忆 $\mathrm{T}$ 细 胞(effector memory $\mathrm{T}$ cells, $\left.\mathrm{T}_{\mathrm{EM}}\right)^{[1]}$. $\mathrm{T}_{\mathrm{CM}}$ 表达CD45RO, $\mathrm{CCR} 7$ 和 CD62L, 使其可以通过高内皮静脉进入次级器 官. 再次接触抗原刺激时, $\mathrm{T}_{\mathrm{CM}}$ 主要表达IL-2, 并能迅速 分裂, 补充周围器官中的效应 $\mathrm{T}$ 细胞. $\mathrm{T}_{\mathrm{EM}}$ 表达 CD45RO，不表达CCR7和CD62L，而表达其他趋化因 子受体和黏附分子, 使其游走至感染组织 ${ }^{[2]}$. 与 $\mathrm{T}_{\mathrm{CM}}$ 相 比, 在 $\mathrm{T}$ 细胞抗原受体(T-cell receptor, TCR)刺激后, $\mathrm{T}_{\mathrm{EM}}$ 增殖较慢, 但能迅速发生免疫应答. 2001年有文献报道 小鼠流感模型中存在一群长期驻留于肺组织的 $\mathrm{CD} 8^{+}$
$\mathrm{T}$ 细胞. 此外, 李斯特菌和水疱性口炎病毒感染后 ${ }^{[3]}$, 抗 原特异性 $\mathrm{CD}^{+} \mathrm{T}$ 细胞可迁移至非淋巴结组织并长期

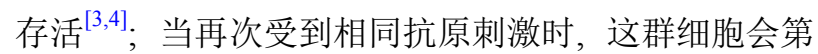
一时间激活, 对抗感染, 被命名为组织驻留记忆 $\mathrm{T}$ 细胞 (tissue resident memory $\mathrm{T}$ cells, $\left.\mathrm{T}_{\mathrm{RM}}\right)^{[3,4]}$. 已经证实 $\mathrm{CD} 8^{+}$ $\mathrm{T}_{\mathrm{RM}}$ 细胞存在于肺、皮肤、阴道和呼吸系统、泌尿系 统等组织中, 发挥细胞毒性作用 ${ }^{[5]}$. 2011年, Farber课题 组 ${ }^{[6]}$ 在小鼠嵌合体实验中发现, 肺部 $C D 4^{+} \mathrm{T}_{\mathrm{RM}}$ 不仅有 组织趋向性, 而且其表型不同于脾脏和外周循环的 $\mathrm{CD}^{+} \mathrm{T}$ 细胞(图1). 此外, $\mathrm{CD}^{+} \mathrm{T}_{\mathrm{RM}}$ 可长期驻留在肺实 质中, 抵抗结核分枝杆菌感染, 快速清除细菌. 研究表 明, 几乎所有组织, 包括肺、肝、胰腺、淋巴组织、泌

引用格式: 吴琼丽, 康双朋, 吴长有. 组织驻留记忆T细胞与肿瘤研究进展. 中国科学: 生命科学, 2020, 50: 1032-1041

Wu Q L, Kang S P, Wu C Y. The progress of tissue resident memory T-cells against tumors (in Chinese). Sci Sin Vitae, 2020, 50: 1032-1041, doi: $10.1360 /$ SSV-2020-0024 


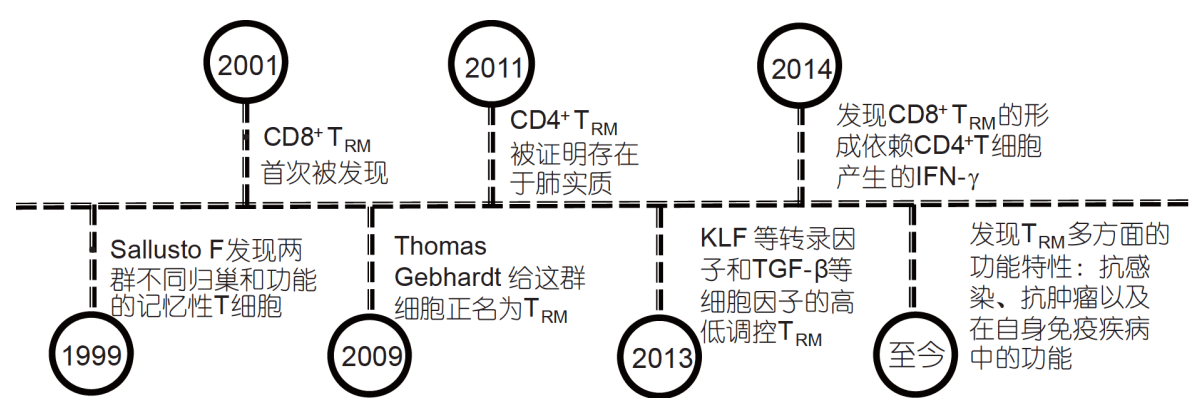

图 $1 \mathrm{~T}_{\mathrm{RM}}$ 的研究进展

Figure 1 The progress of tissue resident memory $\mathrm{T}$ cells

尿和生殖器黏膜、胃肠道(胃、空肠、回肠和结肠)、 骨髓和大脑等组织均存在 $\mathrm{T}_{\mathrm{RM}}$ 细胞 ${ }^{[7 \sim 13]}$. 事实上, 许多 其他的白细胞谱系也具有驻留能力, 包括固有淋巴细 胞(innate lymphoid cells, ILCs)、巨潄细胞 (macrophage, $M \varphi$ )、自然杀伤细胞(natural killer cell, NK)、 记忆 $B$ 细胞和浆细胞 ${ }^{[14]}$. 目前, 关于 $T_{R M}$ 细胞的发生和 维持机制尚不明确. 有文献报道, KLF和KLF2 $2^{[15]}$ 等转 录因子可调控 $T_{R M}$ 的组织驻留能力, TGF- $\beta$ 和IFN $-\alpha$ 等 细胞因子促进了 $\mathrm{T}_{\mathrm{RM}}$ 的分化 ${ }^{[16,17]} . \mathrm{CD} 4^{+} \mathrm{T}$ 细胞辅助 $\mathrm{CD}^{+} \mathrm{T}_{\mathrm{RM}}$ 的形成, $\mathrm{CD}^{+} \mathrm{T}$ 细胞产生的IFN $-\gamma$ 促使 $\mathrm{CD} 8^{+}$ $\mathrm{T}$ 细胞进入皮肤和黏膜等部位，分化成具有杀伤功能 的 $\mathrm{CD}^{+} \mathrm{T}_{\mathrm{RM}}$ 细胞 ${ }^{[18-20]}$. $\mathrm{T}_{\mathrm{RM}}$ 在抗感染、抗肿瘤以及在 自身免疫性疾病中发挥重要的功能 ${ }^{[21]}$.

\section{$1 \mathrm{~T}_{\mathrm{RM}}$ 细胞的分化与调控}

$\mathrm{T}_{\mathrm{RM}}$ 细胞通常存在于病原入侵部位, 能够精准、 快速高效地进行免疫应答反应. 目前, $\mathrm{T}_{\mathrm{RM}}$ 细胞的分化 主要有3种模式(图2). (i) 当病原入侵皮肤上皮层时, 树突状细胞 (dendritic cells, DC)和/或BATF3 ${ }^{+}$DC细胞 均可捕获、识别、加工、处理抗原, 进入淋巴组织, 提 呈给初始 $T$ 细胞 (naive $\mathrm{T}$ cell, $\mathrm{T}_{\mathrm{N}}$ ), 诱导 $\mathrm{T}_{\mathrm{N}}$ 细胞的活化、 增殖和分化. 研究表明, 经DC细胞提呈后, $\mathrm{T}_{\mathrm{N}}$ 细胞主要 分化为 $T_{C M}, T_{E M}$ 与 $T_{E F F}$ 细胞, 而经BATF $3^{+}$DC提呈后, $T_{N}$ 与 $T_{C M}$ 细胞分化为 $T_{R M}$ 前体细胞 (precursors $T_{R M}$ cells, $\mathrm{pT}_{\mathrm{RM}}$ ), 随后 $\mathrm{pT}_{\mathrm{RM}}$ 进入血液, 经循环进入组织. 在巨噬细 胞释放的IFN- $\alpha$ 作用下表达CD69, 到达上皮层, 在 TGF- $\beta$ 的作用下表达CD103，促使 $\mathrm{T}_{\mathrm{RM}}$ 细胞分化. CD69 与 $S 1 P R 1$ 的结合, 使 $T$ 淋巴细胞能够长期存在于外周组 织并分化发育为 $\mathrm{T}_{\mathrm{RM}}$. (ii) 在循环系统中, 初始 $T$ 细胞
在淋巴结内接受抗原提呈细胞的信号, 分化为 $\mathrm{T}_{\mathrm{EFF}}$, 在 免疫应答之后, 大部分 $\mathrm{T}_{\mathrm{EFF}}$ 调亡, 部分 $\mathrm{T}_{\mathrm{EFF}}$ 分化成 $\mathrm{T}_{\mathrm{CM}}$ 与 $T_{\mathrm{EM}}$ 细胞. 少部分 $\mathrm{T}_{\mathrm{EFF}}$ 细胞在选择素、趋化因子、整 合素的联合作用下在血液、循环系统中迁移到局部, 识别该抗原, 瞬时降低转录因子KLF2. 除CD69外, KLF2 是T淋巴细胞表达S1PR1 所必需的转录因子 ${ }^{[18]}$, 当T淋巴细胞进入外周非淋巴组织后即下调KLF2的表 达, 从而使S1PR1的表达下调，同时KLF2表达的下调 使 CCR7 的表达也下调, 而 $\mathrm{CCR} 7$ 是 $\mathrm{T}$ 细胞撤出外周非 淋巴组织所必需的趋化因子. 综上, KLF2是调节T细 胞转运的关键转录因子之一, KLF2及其靶基因 SIPR1 的下调促进了 $\mathrm{T}$ 细胞在组织中的驻留 ${ }^{[22]}$. 而 $\mathrm{S} 1 \mathrm{PR} 1$ 与 细胞 $\mathrm{C}$ 型凝集素 $\mathrm{CD} 69$ 表达相拮抗, 促使 $\mathrm{CD} 69$ 上调. 同 时, 局部产生的TGF- $\beta$, TNF- $\alpha$, IFN和IL-33均参与 $\mathrm{CD} 103$ 的表达. 在病原清除后, $\mathrm{T}_{\mathrm{RM}}$ 细胞常驻上皮层不 再参与外周循环. 研究显示, 小鼠与人类KLF2和 $\mathrm{S} 1 \mathrm{PR} 1$ 在组织中的 $\mathrm{CD}^{+} \mathrm{T}_{\mathrm{RM}}{ }^{[11,23-25]}, \mathrm{CD}^{+} \mathrm{T}_{\mathrm{RM}}{ }^{[26]}$ 以及 $\mathrm{NK} \mathrm{T}_{\mathrm{RM}}$ 细胞中普遍下调, 细胞因子 TGF- $\beta$ 和IL-15均参 与了这一过程 ${ }^{[1,25]}$. (iii) 在小鼠和人类急性病原感染 小肠后，循环系统中的 $\mathrm{T}_{\mathrm{EFF}}$ 细胞瞬时上调 $\alpha 4 \beta 7$ 整合素, 促进其向小肠黏膜移动, 进入肠道后 $\alpha 4 \beta 7$ 迅速下调, 这种 $\alpha 4 \beta 7$ 的下调伴随着 $\alpha$ e $\beta 7$ 整合素 $(\alpha \mathrm{e}$ 也被称为 $\mathrm{CD} 103$ )和 $\mathrm{CD} 69$ 的原位上调, 效应 $\mathrm{CD} 8^{+} \mathrm{T}$ 细胞在进入 肠道后失去了再出肠道的能力, 持续分泌granzyme B 对靶细胞进行杀伤. 除以上 3 种主要分化途径外, 肺单 核细胞与肺组织中的效应 T细胞相互作用并参与驱动 $\mathrm{T}_{\mathrm{RM}}$ 的分化 ${ }^{[27]}$; 肺泡巨喍细胞也参与调控 $\mathrm{T}_{\mathrm{RM}}$ 的早期分 化 ${ }^{[27]}$; 此外, 在传染病模型中, 新的证据表明, 单核细 胞来源的抗原提呈细胞(monocyte-derived antigen-presenting cell, MoAPC)在 $\mathrm{T}_{\mathrm{RM}}$ 细胞的分化、定位以及存 


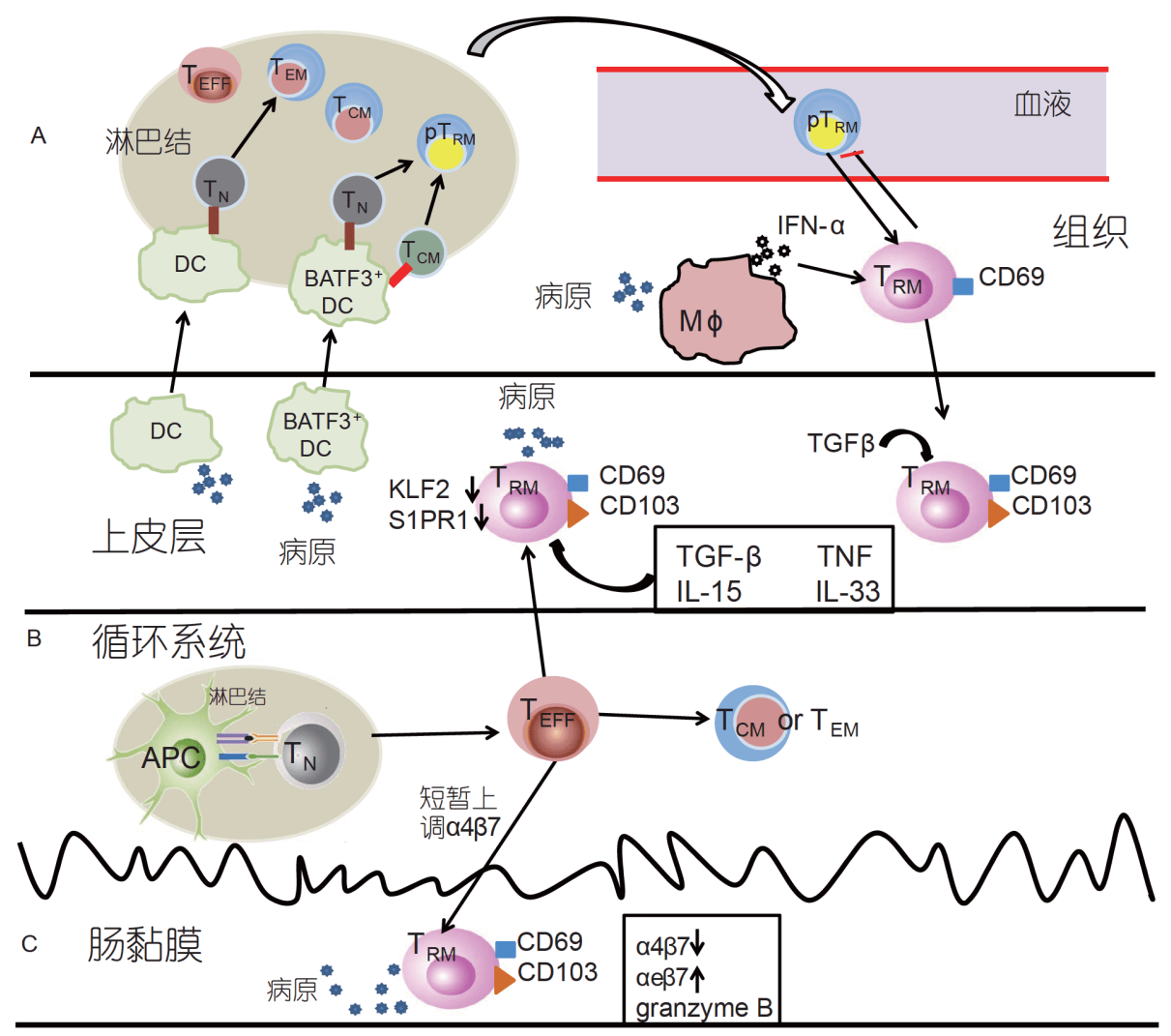

图 $2 \mathrm{~T}_{\mathrm{RM}}$ 细胞 3 种分化途径. A: DC和/或BATF3 ${ }^{+} \mathrm{DC}$ 识别、加工、处理抗原, 进入淋巴结, 诱导初始 $\mathrm{T}$ 细胞的活化、增殖和分

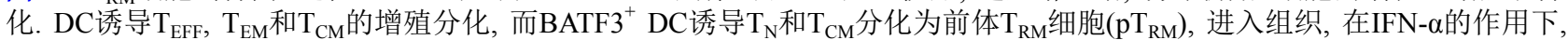
$\mathrm{T}_{\mathrm{RM}}$ 表达CD69, 进入到上皮层, 在TGF- $\beta$ 的作用下表达CD103. B: 在淋巴结中, $\mathrm{T}_{\mathrm{N}}$ 分化成 $\mathrm{T}_{\mathrm{EFF}}$ 后, 部分进入上皮组织中, 识别抗 原, 瞬时下调转录因子KLF2, 导致S1PR1下调, 促使CD69上调. 同时, 局部产生的TNF, IFN- $\alpha$ 和IL-33细胞因子诱导细胞表达 $\mathrm{CD} 103$, 使得细胞驻留在组织中. $\mathrm{C}$ : 病原入侵肠黏膜后, $\mathrm{T}_{\mathrm{EFF}}$ 快速上调 $\alpha 4 \beta 7$ 整合素水平, 促进其向肠黏膜移动, 当进入肠黏膜组 织后, $\alpha 4 \beta 7$ 急速下调, $\mathrm{T}_{\mathrm{RM}}$ 表达CD69和CD103, 驻留在肠黏膜组织中

Figure 2 The differentiation of tissue resident memory T cells in three ways. A: DC and/or BATF $3^{+}$DC recognize, process, present antigens and induce the activation, proliferation, and differentiation of $\mathrm{T}_{\mathrm{N}}$ in lymph nodes. $\mathrm{DC}$ induces the proliferation and differentiation of $\mathrm{T}_{\mathrm{EFF}}, \mathrm{T}_{\mathrm{EM}}$, and $\mathrm{T}_{\mathrm{CM}}$, while BATF3 ${ }^{+}$DC induces $T_{N}$ and $T_{C M}$ to differentiate into precursors $T_{R M}$ cells $\left(p T_{R M}\right)$, and enter the tissues where $T_{R M}$ expresses $C D 69$ under the role of IFN- $\alpha$. Afterwards, $T_{\mathrm{RM}}$ migrate to the epithelial, and expresses CD103 under the action of TGF- $\beta$. B: After $\mathrm{T}_{\mathrm{N}}$ differentiate into $\mathrm{T}_{\mathrm{EFF}}$ in lymph nodes, some of them enter the epithelial tissues to recognize antigens, and the transcription factor KLF2 is reduced instantaneously, leading to the down-regulation of S1PR1 and the up-regulation of CD69. At the same time, TNF, IFN- $\alpha$ and IL-33 induced the cells to express CD103 and reside in the tissues. C: After the pathogen invaded the intestinal mucosa, $\mathrm{T}_{\mathrm{EFF}}$ rapidly upregulated $\alpha 4 \beta 7$ and were promoted to migrate to the intestinal mucosa. When it entered the intestinal mucosa, $\alpha 4 \beta 7$ was rapidly down-regulated, $\mathrm{T}_{\mathrm{RM}}$ cells expressed CD69 and CD103 and resided in the intestinal mucosa

活方面提供趋化因子和细胞因子并起关键作用。小鼠 生殖器感染疱疹病毒时, $\mathrm{CD}^{+}{ }^{+} \mathrm{T}_{\mathrm{RM}}$ 表达的IFN- $\gamma$ 诱导 $\mathrm{M} \varphi$ 表达CCL5,CCL5 ${ }^{+} \mathrm{M} \varphi$ 帮助这些 $\mathrm{T}_{\mathrm{RM}}$ 驻留在组织中; 肠内感染耶尔森氏杆菌时，CCR2依赖的 $\mathrm{M} \varphi$ 产生的细 胞因子IFN- $\alpha / \beta$ 和IL-12调节 $C D 8^{+} \mathrm{T}_{\mathrm{RM}}$ 的分化和维持. 然而, MoAPC在调控这些细胞维持及存活方面的机制 尚不清楚 ${ }^{[28]}$, 以及感染解除后MoAPC发生了什么, 它 们在 $\mathrm{T}_{\mathrm{RM}}$ 形成和维持的小环境起到的作用，也仍需进 一步研究.
T-bet和Eomes 是 $\mathrm{T}_{\mathrm{EM}}$ 和 $\mathrm{T}_{\mathrm{CM}}$ 的关键调控因子 ${ }^{[26]}$. T-bet和Eomes在 $\mathrm{CD} 8^{+} \mathrm{T}_{\mathrm{RM}}$ 中均下调 $\mathrm{CD} 103$ 在皮肤、肠 道、肺和大脑中的表达 ${ }^{[29,30]}$, 抑制皮肤和肺中 $\mathrm{T}_{\mathrm{RM}}$ 的形 成, 而组织微环境中存在的TGF- $\beta$ 下调T-bet和Eomes的 表达, 促进 $\mathrm{T}_{\mathrm{RM}}$ 分化 ${ }^{[31]}$.

\section{$2 \mathbf{T}_{\mathrm{RM}}$ 的种类和功能}

研究表明, 除CD8 ${ }^{+} \mathrm{T}_{\mathrm{RM}}$, 还有 $\mathrm{CD} 4^{+} \mathrm{T}_{\mathrm{RM}}$, Treg $\mathrm{T}_{\mathrm{RM}}$, 
$\gamma \delta \mathrm{T} \mathrm{T}_{\mathrm{RM}}$ 以及 $\mathrm{NKT} \mathrm{T}_{\mathrm{RM}}$ 都在抗感染、抗肿瘤以及自身 免疫性疾病的发生发展中发挥重要作用 ${ }^{[32]} . \mathrm{CD}^{+} \mathrm{T}_{\mathrm{RM}}$ 在肺、皮肤、肠道等组织中长期驻留，当再次遭遇相 同抗原时可快速增殖，发挥抗感染作用. 近期多研究 表明, 肿瘤组织中浸润有大量 $\mathrm{CD} 8^{+} \mathrm{T}_{\mathrm{RM}}$ 细胞, 浸润的 细胞数量与肿瘤的分期以及预后息息相关. 肿瘤浸润 的 $\mathrm{CD}^{+} \mathrm{T}_{\mathrm{RM}}$ 细胞高表达颗粒酶B、IFN- $\gamma$ 和TNF- $\alpha$, 发 挥杀伤功能 ${ }^{[33]} \cdot \mathrm{CD}^{+} \mathrm{T}_{\mathrm{RM}}$ 细胞除发挥抗感染功能外, 还可促进 $\mathrm{CD}^{+} \mathrm{T}_{\mathrm{RM}}$ 细胞的产生. Laidlaw等人 ${ }^{[19]}$ 的研 究表明, 小鼠感染流感病毒后会清除 $\mathrm{CD} 4^{+} \mathrm{T}$ 细胞, 缺 $\sum \mathrm{CD} 4^{+} \mathrm{T}$ 细胞辅助的 $\mathrm{CD} 8^{+} \mathrm{T}$ 细胞不能上调 $\mathrm{CD} 69$, CD103的表达, 无法驻留在气道上皮内, 不能形成 $\mathrm{CD}^{+} \mathrm{T}_{\mathrm{RM}}$ 细胞. 在阴道黏膜感染过程中, $\mathrm{CD} 4^{+} \mathrm{T}_{\mathrm{RM}}$ 细 胞产生IFN- $\gamma$, 可通过诱导趋化因子CXCL9, CXCL10 和CXCL11的表达，趋化CD $8^{+} \mathrm{T}_{\mathrm{RM}}$ 进入阴道黏膜组 织 ${ }^{[34]} . \gamma \delta \mathrm{T} \mathrm{T}_{\mathrm{RM}}$ 主要聚集于皮肤、小肠、女性生殖道 等黏膜组织部位，在黏膜免疫中发挥重要作用 ${ }^{[17]}$. 皮 肤组织内驻留的 $\gamma \delta \mathrm{T}$ 细胞参与皮肤创伤的愈合 ${ }^{[35]}$. 尹 芝南教授课题组 ${ }^{[36]}$ 的研究表明, $\gamma \delta \mathrm{T}$ 细胞可通过产生 IFN- $\gamma$ 促进神经损伤的修复. 有趣的是, 脂肪组织驻留 的 $\gamma \delta \mathrm{T}$ 细胞在促炎因子 IL-1 $\beta$ 和IL-23的诱导下, 迅速产 生IL-17和TNF- $\alpha$, 激活基质细胞分泌IL-33. IL-33可作 用于脂肪组织中 $\mathrm{ST}^{+}{ }^{+} \mathrm{Treg}$ 细胞, 进而调控人和小鼠的

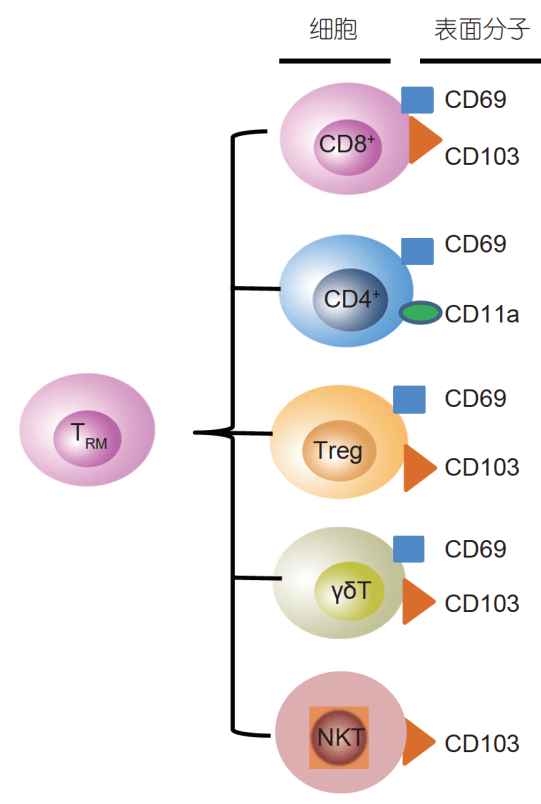

图 $3 \mathrm{~T}_{\mathrm{RM}}$ 的不同种类及功能

Figure 3 Subsets and functions of the tissue resident memory T cells
体温. $\gamma \delta \mathrm{T}$ 细胞缺陷或者IL-17A $\mathrm{A}^{-/}$的小鼠在寒冷时无 法自发地调节体温. 本课题组 ${ }^{[37]}$ 研究发现, 人和小鼠 子宫体内都存在一群组织驻留记忆 $\gamma \delta \mathrm{T}$ 细胞, 高表达 IL-17，受雌性激素的调控. 在自身免疫性疾病以及慢 性感染炎症部位, 还存在Treg $\mathrm{T}_{\mathrm{RM}}$ 调节性的组织驻留 细胞, 其主要作用是调节免疫反应, 维持机体稳态. 此 外, 研究表明, 组织中存在的 $N K \mathrm{~T}_{\mathrm{RM}}$ 细胞可识别 $\mathrm{CD} 1 \mathrm{~d}$ 糖脂抗原, 在肿瘤监测及控制病毒和细菌感染中发挥 作用 ${ }^{[15,35]}$ (图3). 研究表明, 环境蛋白质亦可诱导 $\mathrm{T}_{\mathrm{RM}}$ 细 胞产生, 累积的 $\mathrm{T}_{\mathrm{RM}}$ 细胞一旦被激活可造成严重的慢 性炎症反应，对机体造成损坏，这可能是某些自身免 疫性疾病的发病原因. 在小鼠和人体中的研究发现, 过敏性接触皮炎由局部驻留的 $\mathrm{T}_{\mathrm{RM}}$ 细胞介导 ${ }^{[38]}$.

\section{$3 \mathrm{~T}_{\mathrm{RM}}$ 的抗肿瘤作用}

在免疫应答过程中， $\mathrm{T}$ 细胞被激活从而增殖、分 化, 部分 $\mathrm{T}$ 细胞被保留在外周组织中, 而不再参与血液 循环 ${ }^{[39]}$. 这些组织驻留的 $\mathrm{CD} 8^{+}$记忆 $\mathrm{T}$ 细胞在屏障表面 和非淋巴器官中巡逻, 保护机体免受病毒和细菌感染 以及肿瘤的发生. 最近的研究表明, 肿瘤免疫治疗已 成为继手术、放疗、化疗、靶向治疗后最有发展前景 的治疗方法 ${ }^{[40]}$. $\mathrm{T}_{\mathrm{RM}}$ 细胞在阻止实体肿瘤的发展和转

\begin{tabular}{l} 
功能 \\
\hline 在再感染情况下快速发挥免疫保护, 对抗 \\
感染, 起到细胞毒性作用; 且抗肿瘤, 与 \\
肿瘤分期和预后密切相关. \\
在对抗结核分枝杆菌感染中提供了更好的 \\
保护, 它们在快速清除继发性感染的细菌 \\
中发挥重要作用. \\
促进组织修复、维持组织免疫稳态, 还可 \\
抑制多种慢性感染引起的炎症反应和损伤. \\
在体内组织平衡、修复及免疫防御中起 \\
重要作用. \\
\end{tabular}


移等方面起重要作用．恶性肿瘤的发生、发展常经过 转化、克隆性增生和演进. 大多数实体肿瘤患者的死 亡不是由于原发肿瘤的生长引起，而是由于肿瘤细胞 转移、逃逸免疫监视导致, 因此免疫监视对保护肿瘤 免疫起关键作用 ${ }^{[41]}$. 肿瘤进展中 $\mathrm{T}_{\mathrm{RM}}$ 细胞的浸润程度 与临床的分期和治疗预后有极大的关系. 肿瘤中 $\mathrm{CD} 8^{+}$ $\mathrm{T}_{\mathrm{RM}}$ 浸润程度高、肿瘤分期较低, 患者预后更好 ${ }^{[5]}$.

$\mathrm{CD} 8^{+} \mathrm{T}_{\mathrm{RM}}$ 具有强大的细胞毒性活性. $\mathrm{CD} 8^{+}$ $\mathrm{CD} 103^{+}$TIL高表达颗粒酶A、B和穿孔素 ${ }^{[33,42]}$. 此外, 越来越多的研究表明, $\mathrm{T}_{\mathrm{RM}}$ 细胞常积聚于肿瘤中, 尤其 是上皮来源的肿瘤 ${ }^{[43]} . \mathrm{CD} 8^{+} \mathrm{CD} 103^{+} \mathrm{T}$ 细胞直接杀伤上 皮来源的肿瘤细胞. 同时, 激活 $\mathrm{CD} 103$ 可直接使 $\mathrm{CD}^{+} \mathrm{T}$ 细胞释放穿孔素和颗粒酶 $\mathrm{B}$ ，增强抗肿瘤免疫应 答 ${ }^{[33,42]}$ (图4). $\mathrm{CD} 103^{+}$淋巴细胞亚群数量增加与患者病 情改善和生存期相关. 膀胱癌中 $\mathrm{CD} 103^{+}$肿瘤浸润淋巴 细胞增多也与预后良好相关 ${ }^{[44]}, \mathrm{CD} 103^{+} \mathrm{T}$ 细胞的密度 与肿瘤大小成反比. 最近的研究表明, CD103整合素可 作为乳腺癌 ${ }^{[45]}$ 和肺癌 ${ }^{[43]}$ 预后良好的生物标志物. $\mathrm{CD} 8^{+}$ $\mathrm{CD}_{103^{+}} \mathrm{T}$ 细胞已成为很多肿瘤疾病(包括口腔、卵 巢、肺、子宫内膜和乳腺癌)生存的预测标志物 ${ }^{[46-48]}$.
近来研究表明，肿瘤组织中富集的 $\mathrm{T}_{\mathrm{RM}}$ 细胞抑制 性免疫检查点(immune checkpoints, Icps)的表达特别 丰富. 有文献指出, $\mathrm{T}_{\mathrm{RM}}$ 是针对Icps新疗法的主要靶点, 例如程序性死亡-1途径. 最近的研究表明, 在非循环的 情况下, $\mathrm{T}_{\mathrm{RM}}$ 细胞的组织限制也可能导致单个转移灶 内 $\mathrm{T}_{\mathrm{RM}}$ 细胞的异质性, 最终导致细胞间的多样性, 维持 机体免疫稳态. 在越来越多的病例中, $\mathrm{CD}^{+} \mathrm{T}_{\mathrm{RM}}$ 细胞 已被证明可以介导大体积肿瘤的消退, 导致长期的疾 病缓解 ${ }^{[49]}, \mathrm{CD}^{+} \mathrm{T}_{\mathrm{RM}}$ 细胞是抵抗晚期癌症 ${ }^{[50]}$ 的免疫细 胞. 研究发现, $\mathrm{CD}^{+} \mathrm{T}_{\mathrm{RM}}$ 细胞还可以通过促进抗原提 呈细胞对肿瘤抗原的处理, 增强抗肿瘤免疫 ${ }^{[51]}$.

\section{$4 \quad \mathbf{T}_{\mathrm{RM}}$ 在制备抗肿瘤疫苗中的作用}

免疫治疗是癌症治疗的热点之一, 可以追溯到 1891年的Coley疫苗 ${ }^{[52]}$. 近年来的研究表明, 肿瘤浸润 性 $\mathrm{T}$ 细胞主要为 $\mathrm{T}_{\mathrm{RM}}$ 细胞, 为免疫治疗提供了新的潜在 靶点 ${ }^{[53]}$. Ganesan等人 ${ }^{[43]}$ 对头颈部肿瘤患者的研究表 明, 如果有 $\mathrm{T}_{\mathrm{RM}}$ 基因的表达, 抗肿瘤的效果会更好. 肿 瘤组织中 $\mathrm{T}_{\mathrm{RM}}$ 细胞密度高的患者存活时间明显延长,

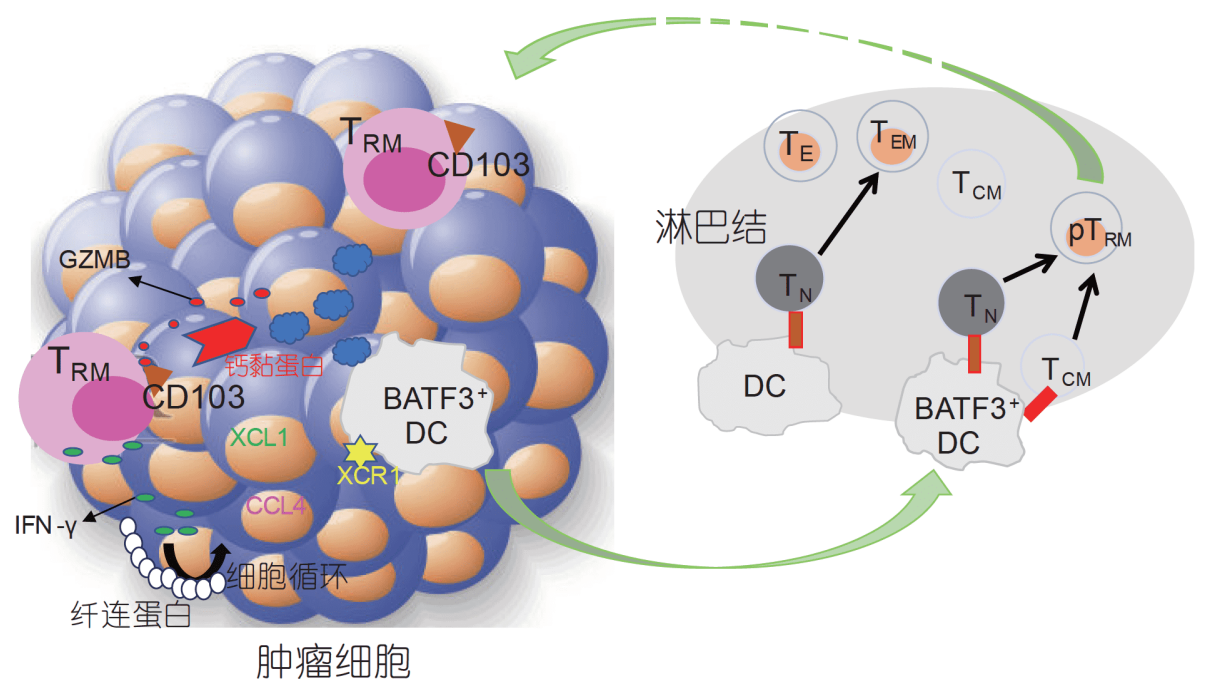

图 $4 \mathrm{CD} 103^{+} \mathrm{T}_{\mathrm{RM}}$ 细胞控制肿瘤细胞生长和转移的机制. $\mathrm{T}_{\mathrm{RM}}$ 细胞表达 CD103分子，识别、结合肿瘤细胞表面的钲黏蛋白分 子, 产生颗粒酶B(GZMB), 直接杀伤肿瘤细胞. 同时也产生IFN- $\gamma$, 抑制肿瘤细胞的生长, 诱导纤连蛋白产生, 限制肿瘤细胞逃 逸. $\mathrm{T}_{\mathrm{RM}}$ 细胞也能产生趋化因子 $\mathrm{CCL} 4, \mathrm{XCL} 1$, 募集XCR $1^{+} \mathrm{BATF} 3^{+} \mathrm{DC}$ 细胞, 摄取提呈死亡肿瘤细胞碎片, 游走到引流淋巴结内, 诱导 $\mathrm{T}_{\mathrm{N}}$ 细胞分化成 $\mathrm{T}_{\mathrm{E}}$ 细胞和 $\mathrm{p}_{\mathrm{RM}}$ 细胞, 补充肿瘤内 $\mathrm{T}_{\mathrm{RM}}$ 细胞数量, 维持肿瘤免疫, 限制肿瘤细胞生长

Figure 4 The mechanism of $\mathrm{CD}_{103^{+}}$tissue resident memory $\mathrm{T}$ cells in the control of the growth and metastasis of tumor cells. $\mathrm{T}_{\mathrm{RM}}$ cells express CD103 molecule, which recognizes and binds the calcmucin molecule on the surface of tumor cells and produces granzyme-B to directly kills tumor cells. It also produces IFN- $\gamma$, which can inhibit the growth of tumor cells and induce the production of fibronectin to limits the escape of tumor cells. $\mathrm{T}_{\mathrm{RM}}$ cells can also produce chemokines CCL4 and XCL1, leading to recruitment of XCR $1^{+} \mathrm{Batf}^{+} \mathrm{DCs}$ and activate T cells. The uptake of antigens from dying tumor cells by XCR1 ${ }^{+} \mathrm{Batf3}^{+} \mathrm{DCs}$ can result in the priming of $\mathrm{T}_{\mathrm{N}}$ or $\mathrm{T}_{\mathrm{CM}}$ cells in draining lymph nodes to supply the number of $\mathrm{T}_{\mathrm{RM}}$ cells in the tumors and limit the growth of tumor cells 
表明 $\mathrm{T}_{\mathrm{RM}}$ 细胞在预防肿瘤复发方面发挥了关键作用 ${ }^{[54]}$. 此外，肿瘤逃逸的机制与免疫检查点分子过度表达或 功能过强相关，其过度表达或功能过强都会导致免疫 功能受到抑制, 使机体的免疫力低下, 肿瘤易发生. 由 于 $\mathrm{T}_{\mathrm{RM}}$ 细胞在局部免疫中的作用, 制备疫苗, 接种后诱 导 $\mathrm{T}_{\mathrm{RM}}$ 细胞, 对预防和抗肿瘤十分重要. 目前已有研究 证明, 经修饰后的牛痘病毒载体疫苗能激活鼻黏膜相 关淋巴组织中的 $\mathrm{T}_{\mathrm{RM}}$ 细胞并促进增殖和分裂, 使其数 目增加, 表现出效应记忆 T细胞特征; 当再次识别相同 抗原时, 释放颗粒酶B和促炎细胞因子, 杀伤靶细胞 ${ }^{[55]}$. 此外, 疫苗接种的途径对促进 $\mathrm{T}_{\mathrm{RM}}$ 生成至关重要. 直接 在黏膜中接种的肿瘤疫苗与经肌肉或皮下接种途径的 疫苗相比, 常导致黏膜肿瘤中 $\mathrm{T}_{\mathrm{RM}}$ 的形成增强 ${ }^{[20]}$. 在头 颈癌的临床前研究发现, 鼻内接种志贺毒素 $\mathrm{B}$ 亚基, 可 诱导局部 $\mathrm{T}_{\mathrm{RM}}$ 生成并抑制肿瘤生长 ${ }^{[55]}$. 然而, 截至目前, 关于 $\mathrm{T}_{\mathrm{RM}}$ 在肿瘤疫苗接种后, 对肿瘤免疫监测中的作 用, 还缺乏直接证明 ${ }^{[50,55]}$.

\section{$5 \mathrm{~T}_{\mathrm{RM}}$ 的调节性作用}

$\mathrm{T}_{\mathrm{RM}}$ 细胞不仅具有抗感染和抗肿瘤的作用, 还具 有免疫调节功能，在维持机体免疫平衡、自身免疫性 疾病的发生发展中发挥重要作用 ${ }^{[56]}$. 常见的发挥免疫 调节功能的 $\mathrm{T}_{\mathrm{RM}}$ 主要是Treg $\mathrm{T}_{\mathrm{RM}}$ 细胞. 研究表明, 在皮 肤、肺、肌肉、脂肪、关节等组织部位都存在组织驻 留的Treg细胞, 且具有组织特异性, 不同组织的 Treg细 胞具有不同的免疫特征，通过不同的机制发挥免疫调 节功能 ${ }^{[57]}$ (图5). 脂肪组织中存在的 Treg细胞, 可占 $\mathrm{CD}^{+} \mathrm{T}$ 细胞的 $40 \% \sim 60 \%$, 表达高水平的IL-10. IL-10
抑制促炎因子和趋化因子的表达，从而中断肥胖相关 代谢障碍的发展 ${ }^{[58]}$. 此外, 组织驻留的Treg细胞在肌 肉组织再生、骨骼吸收和重塑、抑制自身免疫性疾病 中具有重要意义 $义^{[59-61]}$. 研究发现, $\mathrm{CD} 103^{+} \mathrm{T}_{\mathrm{RM}}$ 亦可发 挥免疫调节功能, 但 $\mathrm{CD} 103^{+} \mathrm{CD} 25^{+}$双阳性 $\mathrm{T}_{\mathrm{RM}}$ 细胞比 $\mathrm{CD} 103^{+} \mathrm{CD} 25^{-}$单阳性细胞的免疫调节功能更强大, $\mathrm{CD}_{103^{+}} \mathrm{T}_{\mathrm{RM}}$ 高表达CTLA-4, ICOS, FAS, GITR等免疫 抑制分子 ${ }^{[62]}$. 此外, 肿瘤浸润的 Treg $\mathrm{T}_{\mathrm{RM}}$ 的多少与肿瘤 预后负相关，有充分的证据表明，Treg的耗竭或抑制 Treg功能可以增强抗肿瘤作用 ${ }^{[63]}$, 肿瘤组织内浸润的 调节性 T细胞可通过产生细胞因子IL-10, TGF- $\beta$ 和 IL-35抑制效应T细胞的功能 ${ }^{[64]}$. 本课题组先前的研 究 ${ }^{[37]}$ 也发现, 小鼠肺、子宫和阴道等组织的 $\mathrm{T}_{\mathrm{RM}}$ 细胞 在体外刺激后, $\mathrm{CD}_{103}{ }^{+}$细胞比 CD $103^{-}$细胞表达更低 水平的IFN- $\gamma$ 和IL-17.

\section{6 展望}

$\mathrm{T}_{\mathrm{RM}}$ 细胞表达CD69和/或CD103，位于病原菌入侵 的初始部位, 为皮肤、生殖道等周围屏障组织的病原 菌入侵提供一线防御, 在感染免疫中发挥快速的防御 反应，同时参与肿瘤、变态反应性和自身免疫性疾病 的调控, 是疫苗研究的基础. $\mathrm{T}_{\mathrm{RM}}$ 为疫苗开发创造了条 件, 需研究的问题包括疫苗接种路线, 抗原的持久性, 以及免疫刺激信号如何优先建立 $\mathrm{T}_{\mathrm{RM}}$ 系统等方面, 并 仍有很大的发现空间. $\mathrm{T}_{\mathrm{RM}}$ 细胞的形成、维持、调控 以及与疾病之间的关系尚不清楚, 需要进一步深入研 究. 在抗肿瘤免疫反应中, $\mathrm{CD}^{+} \mathrm{CD} 103^{+} \mathrm{T}_{\mathrm{RM}}$ 细胞浸润 肿瘤发挥不可替代的作用. CD103 是肿瘤反应性

功能

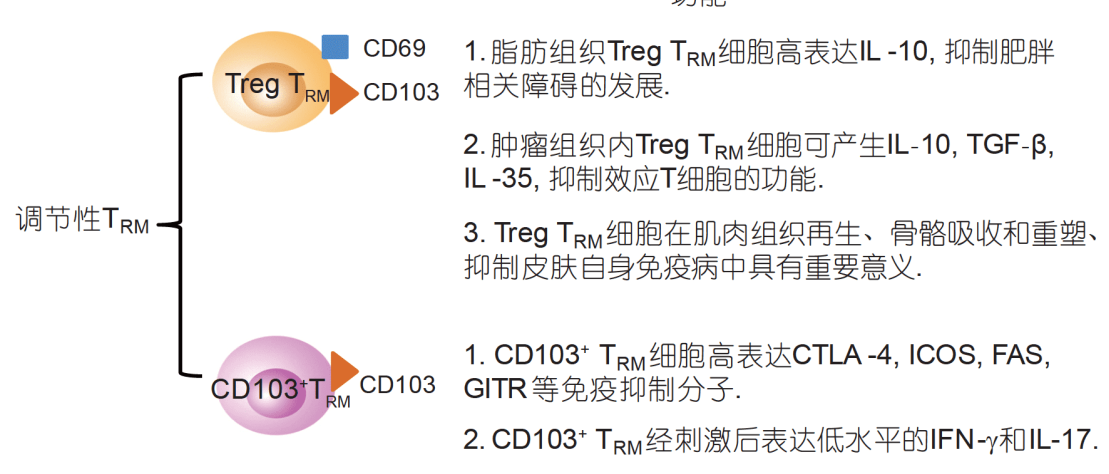

图 5 组织驻留调节性 $\mathrm{T}_{\mathrm{RM}}$ 细胞的种类和功能

Figure 5 The subtypes and functions of tissue resident regulatory tissue resident memory T cells 
$\mathrm{CD}^{+}$肿瘤浸润淋巴细胞的关键生物标志物，可作为肿 瘤预后的预测分子. 此外, 肿瘤浸润 $\mathrm{T}_{\mathrm{RM}}$ 细胞高表达免 疫检查点分子，根据发现的免疫检查点分子并研制调 节这些分子表达或功能的药物有很大的临床应用前 景. 表面标志物CD103在抗肿瘤免疫治疗中尤为重要. 然而, 如何上调 $\mathrm{CD} 8^{+} \mathrm{T}$ 细胞表面CD103的表达, 增加肿 瘤相关 $\mathrm{T}_{\mathrm{RM}}$ 细胞的数量, 降低免疫检查点的表达, 增强 免疫防御, 提高免疫治疗水平, 是抗肿瘤研究的重要方 向. 同时, $\mathrm{T}_{\mathrm{RM}}$ 细胞的驻留特点也为器官移植带来了临
床挑战, $\mathrm{T}_{\mathrm{RM}}$ 的组织驻留和免疫反应迅速强烈特性, 使 得器官移植患者容易发生移植物抗宿主疾病; 此外, 当 $\mathrm{T}_{\mathrm{RM}}$ 细胞识别出无害的抗原或自身抗原时, 它们的激 活会导致机体严重受损，这种损害可能是长期的，也 可能是反复发生的. $\mathrm{CD}^{+} \mathrm{T}_{\mathrm{RM}}$ 细胞最近被证明可以驱 动过敏性气道疾病(在小鼠中模拟哮喘), 并可能导致 屏障部位的延迟型超敏反应。如何靶向清除或抑制供 体器官以及过敏性气道的 $\mathrm{T}_{\mathrm{RM}}$ 细胞, 为临床移植和治 疗服务, 也具有重大意义.

\section{参考文献}

1 Sallusto F, Lenig D, Förster R, et al. Two subsets of memory T lymphocytes with distinct homing potentials and effector functions. Nature, 1999, 401: 708-712

2 Yu S F, Wu C Y. Advances in the study of the immunological characteristics of tissue-resident memory T cells (in Chinese). Chin J Immun, 2017, 33: 1093-1100 [余思菲, 吴长有. 组织定居记忆性T细胞的免疫学特征研究进展. 中国免疫学杂志, 2017, 33: 1093-1100]

3 Hogan R J, Usherwood E J, Zhong W, et al. Activated antigen-specific CD8 ${ }^{+} \mathrm{T}$ cells persist in the lungs following recovery from respiratory virus infections. J Immunol, 2001, 166: 1813-1822

4 Masopust D, Vezys V, Marzo A L, et al. Preferential localization of effector memory cells in nonlymphoid tissue. Science, 2001, 291: 2413-2417

5 Hartana C A, Ahlén Bergman E, Broomé A, et al. Tissue-resident memory T cells are epigenetically cytotoxic with signs of exhaustion in human urinary bladder cancer. Clin Exp Immunol, 2018, 194: 39-53

6 Teijaro J R, Turner D, Pham Q, et al. Cutting edge: Tissue-retentive lung memory CD4 T cells mediate optimal protection to respiratory virus infection. J Immunol, 2011, 187: 5510-5514

7 Booth J S, Toapanta F R, Salerno-Goncalves R, et al. Characterization and functional properties of gastric tissue-resident memory T cells from children, adults, and the elderly. Front Immunol, 2014, 5: 294

8 Okhrimenko A, Grün J R, Westendorf K, et al. Human memory T cells from the bone marrow are resting and maintain long-lasting systemic memory. Proc Natl Acad Sci USA, 2014, 111: 9229-9234

9 Pallett L J, Davies J, Colbeck E J, et al. IL-2high tissue-resident T cells in the human liver: Sentinels for hepatotropic infection. J Exp Med, 2017, 214: $1567-1580$

10 Wong M T, Ong D E H, Lim F S H, et al. A high-dimensional atlas of human T cell diversity reveals tissue-specific trafficking and cytokine signatures. Immunity, 2016, 45: 442-456

11 Woon H G, Braun A, Li J, et al. Compartmentalization of total and virus-specific tissue-resident memory CD8 ${ }^{+} \mathrm{T}$ Cells in human lymphoid organs. PLoS Pathog, 2016, 12: e1005799

12 Swaims-Kohlmeier A, Haaland R E, Haddad L B, et al. Progesterone levels associate with a novel population of CCR $5^{+}$CD38 ${ }^{+}$CD4 T cells resident in the genital mucosa with lymphoid trafficking potential. J Immunol, 2016, 197: 368-376

13 Smolders J, Heutinck K M, Fransen N L, et al. Tissue-resident memory T cells populate the human brain. Nat Commun, 2018, 9: 4593

14 Masopust D, Soerens A G. Tissue-resident T cells and other resident leukocytes. Annu Rev Immunol, 2019, 37: 521-546

15 Preston G C, Feijoo-Carnero C, Schurch N, et al. The impact of KLF2 modulation on the transcriptional program and function of CD8 T cells. PLoS ONE, 2013, 8: e77537

16 Iijima N, Iwasaki A. Tissue instruction for migration and retention of TRM cells. Trends Immunol, 2015, 36: 556-564

17 Mueller S N, Mackay L K. Tissue-resident memory T cells: Local specialists in immune defence. Nat Rev Immunol, 2016, 16: 79-89

18 Glennie N D, Yeramilli V A, Beiting D P, et al. Skin-resident memory CD4 ${ }^{+}$T cells enhance protection against Leishmania major infection. J Exp Med, 2015, 212: 1405-1414

19 Laidlaw B J, Zhang N, Marshall H D, et al. $\mathrm{CD}^{+}{ }^{+} \mathrm{T}$ cell help guides formation of $\mathrm{CD} 103^{+}$lung-resident memory $\mathrm{CD} 8^{+} \mathrm{T}$ cells during influenza 
viral infection. Immunity, 2014, 41: 633-645

20 Nakanishi Y, Lu B, Gerard C, et al. CD8 ${ }^{+}$T lymphocyte mobilization to virus-infected tissue requires CD4 ${ }^{+}$T-cell help. Nature, 2009, 462: 510513

21 Dumauthioz N, Labiano S, Romero P. Tumor resident memory T cells: New players in immune surveillance and therapy. Front Immunol, 2018, 9: 2076

22 Wu C Y. Advances in the study of naive and memory T cells (in Chinese). Curr Immunol, 2005, 25: 353-356 [吴长有. 初始和记忆T细胞的研究 进展. 现代免疫学, 2005, 25: 353-356]

23 Park S L, Mackay L K, Gebhardt T. Distinct recirculation potential of CD69 ${ }^{+} \mathrm{CD}_{103^{-}}$and $\mathrm{CD} 103^{+}$thymic memory CD8 ${ }^{+} \mathrm{T}$ cells. Immunol Cell Biol, 2016, 94: 975-980

24 Mackay L K, Minnich M, Kragten N A M, et al. Hobit and Blimp1 instruct a universal transcriptional program of tissue residency in lymphocytes. Science, 2016, 352: 459-463

25 Skon C N, Lee J Y, Anderson K G, et al. Transcriptional downregulation of S1prl is required for the establishment of resident memory CD8 ${ }^{+} \mathrm{T}$ cells. Nat Immunol, 2013, 14: 1285-1293

26 Iijima N, Iwasaki A. A local macrophage chemokine network sustains protective tissue-resident memory CD4 T cells. Science, 2014, 346: 93-98

27 Dunbar P R, Cartwright E K, Wein A N, et al. Pulmonary monocytes interact with effector T cells in the lung tissue to drive TRM differentiation following viral infection. Mucosal Immunol, 2020, 13: 161-171

28 Chu K L, Batista N V, Girard M, et al. Monocyte-derived cells in tissue-resident memory T cell formation. J Immunol, 2020, 204: 477-485

29 Joshi N S, Cui W, Chandele A, et al. Inflammation directs memory precursor and short-lived effector CD ${ }^{+}$T cell fates via the graded expression of T-bet transcription factor. Immunity, 2007, 27: 281-295

30 Wakim L M, Woodward-Davis A, Liu R, et al. The molecular signature of tissue resident memory CD8 T cells isolated from the brain. J Immunol, 2012, 189: 3462-3471

31 Sojka D K, Plougastel-Douglas B, Yang L, et al. Tissue-resident natural killer (NK) cells are cell lineages distinct from thymic and conventional splenic NK cells. eLife, 2014, 3: e01659

$32 \mathrm{Lu} \mathrm{H} \mathrm{M,} \mathrm{Jiang} \mathrm{J} \mathrm{T,} \mathrm{Lu} \mathrm{B} \mathrm{F.} \mathrm{Biological} \mathrm{characteristics} \mathrm{and} \mathrm{anti-tumor} \mathrm{immunity} \mathrm{function} \mathrm{of} \mathrm{tissue-resident} \mathrm{memory} \mathrm{T} \mathrm{cells} \mathrm{(in} \mathrm{Chinese).} \mathrm{Chin} \mathrm{J}$ Clin Lab Sci, 2018, 36: 628-631 [卢慧敏, 蒋敬庭, 卢斌峰. 组织驻留T细胞的生物学特征及抗肿瘤免疫作用. 临床检验杂志, 2018, 36: 628631]

33 Franciszkiewicz K, Le Floc'h A, Jalil A, et al. Intratumoral induction of CD103 triggers tumor-specific CTL function and CCR5-dependent T-cell retention. Cancer Res, 2009, 69: 6249-6255

34 Nakajima C, Mukai T, Yamaguchi N, et al. Induction of the chemokine receptor CXCR3 on TCR-stimulated T cells: Dependence on the release from persistent TCR-triggering and requirement for IFN- $\gamma$ stimulation. Eur J Immunol, 2002, 32: 1792

35 Mackay L K, Kallies A. Transcriptional regulation of tissue-resident lymphocytes. Trends Immunol, 2017, 38: 94-103

$36 \mathrm{Li} \mathrm{Y}, \mathrm{Wu} \mathrm{Y}$, Zhang C, et al. $\gamma \delta \mathrm{T}$ cell-derived interleukin-17A via an interleukin-1 $\beta$-dependent mechanism mediates cardiac injury and fibrosis in hypertension. Hypertension, 2014, 64: 305-314

37 Kang S P, Wu Q L, Wu C Y. Immunological characteristics of tissue-resident memory T cells in uterine (in Chinese). Immunol J, 2017, 33: 937942 [康双朋, 吴琼丽, 吴长有. 子宫组织定居记忆T细胞的免疫学特征. 免疫学杂志, 2017, 33: 937-942]

38 Zhang Y, Ruiz P. Solid organ transplant-associated acute graft-versus-host disease. Arch Pathol Lab Med, 2010, 134:1220-1224

39 Park S L, Gebhardt T, Mackay L K. Tissue-resident memory T cells in cancer immunosurveillance. Trends Immunol, 2019, 40: 735-747

40 Bai L, Wang J, Tian Z G, et al. Research progress in tissue/organ regional immunity and diseases in China (in Chinese). Sci Sin Vitae, 2019, 49: 220-227 [白丽, 王佳, 田志刚, 等. 我国在组织器官区域免疫特性与疾病领域的研究进展. 中国科学: 生命科学, 2019, 49: 220-227]

41 Gebhardt T, Palendira U, Tscharke D C, et al. Tissue-resident memory T cells in tissue homeostasis, persistent infection, and cancer surveillance. Immunol Rev, 2018, 283: 54-76

42 Floc'H AL, Jalil A, Vergnon I et al. $\alpha \mathrm{E} \beta 7$ integrin interaction with E-cadherin promotes antitumor CTL activity by triggering lytic granule polarization and exocytosis. J Exp Med, 2007, 204: 559-570

43 Ganesan A P, Clarke J, Wood O, et al. Tissue-resident memory features are linked to the magnitude of cytotoxic T cell responses in human lung cancer. Nat Immunol, 2017, 18: 940-950

44 Wang B, Wu S, Zeng H, et al. $\mathrm{CD}_{103}{ }^{+}$tumor infiltrating lymphocytes predict a favorable prognosis in urothelial cell carcinoma of the bladder. J 
Urol, 2015, 194: 556-562

Wang Z Q, Milne K, Derocher H, et al. CD103 and intratumoral immune response in breast cancer. Clin Cancer Res, 2016, 22: 6290-6297

Webb J R, Milne K, Watson P, et al. Tumor-infiltrating lymphocytes expressing the tissue resident memory marker CD103 are associated with increased survival in high-grade serous ovarian cancer. Clin Cancer Res, 2014, 20: 434-444

47 Djenidi F, Adam J, Goubar A, et al. $\mathrm{CD}^{+} \mathrm{CD} 103^{+}$tumor-infiltrating lymphocytes are tumor-specific tissue-resident memory $\mathrm{T}$ cells and a prognostic factor for survival in lung cancer patients. J Immunol, 2015, 194: 3475-3486

Xiao Y, Li H, Mao L, et al. CD103 ${ }^{+} \mathrm{T}$ and dendritic cells indicate a favorable prognosis in oral cancer. J Dent Res, 2019, 98: 1480-1487

Wei S C, Duffy C R, Allison J P. Fundamental mechanisms of immune checkpoint blockade therapy. Cancer Discov, 2018, 8: 1069-1086

Wolchok J D, Saenger Y. The mechanism of anti-CTLA-4 activity and the negative regulation of T-cell activation. Oncol, 2008, 13: 2-9

Menares E, Gálvez-Cancino F, Cáceres-Morgado P, et al. Tissue-resident memory $\mathrm{CD} 8^{+} \mathrm{T}$ cells amplify anti-tumor immunity by triggering antigen spreading through dendritic cells. Nat Commun, 2019, 10: 4401

52 Li D, Wang W. Booming cancer immunotherapy fighting tumors. Sci China Life Sci, 2017, 60: 1445-1449

53 Granier C, Blanc C, Karaki S, et al. Tissue-resident memory T cells play a key role in the efficacy of cancer vaccines. Oncoimmunology, 2017, 6: e1358841

54 Burnet M. Cancer-A biological approach: I. The processes of control. II. The significance of somatic mutation. Br Med J, 1957, 1: 779-786

55 Nizard M, Roussel H, Diniz M O, et al. Induction of resident memory T cells enhances the efficacy of cancer vaccine. Nat Commun, 2017, 8: 15221

56 Ichikawa T, Hirahara K, Kokubo K, et al. CD103hi Treg cells constrain lung fibrosis induced by CD103lo tissue-resident pathogenic CD4 T cells. Nat Immunol, 2019, 20: 1469-1480

57 Prasad S, Hu S, Sheng W S, et al. Tregs modulate lymphocyte proliferation, activation, and resident-memory T-cell accumulation within the brain during MCMV infection. PLoS ONE, 2015, 10: e0145457

58 Cipolletta D, Kolodin D, Benoist C, et al. Tissular Tregs: A unique population of adipose-tissue-resident Foxp $3^{+} \mathrm{CD} 4^{+} \mathrm{T}$ cells that impacts organismal metabolism. Seminars Immunol, 2011, 23: 431-437

59 Weirather J, Hofmann U D W, Beyersdorf N, et al. Foxp $3^{+} \mathrm{CD}^{+}$T cells improve healing after myocardial infarction by modulating monocyte/ macrophage differentiation. Circ Res, 2014, 115: 55-67

60 Zaiss M M, Sarter K, Hess A, et al. Increased bone density and resistance to ovariectomy-induced bone loss in FoxP3-transgenic mice based on impaired osteoclast differentiation. Arthrit Rheumat, 2010, 62: 2328-2338

61 Rosenblum M D, Gratz I K, Paw J S, et al. Response to self antigen imprints regulatory memory in tissues. Nature, 2011, 480: 538-542

62 Chang L Y, Lin Y C, Kang C W, et al. The indispensable role of CCR5 for in vivo suppressor function of tumor-derived CD103 ${ }^{+}$effector/memory regulatory T cells. J Immunol, 2012, 189: 567-574

63 Yan S, Zhang Y, Sun B. The function and potential drug targets of tumour-associated Tregs for cancer immunotherapy. Sci China Life Sci, 2019, 62: 179-186

64 Facciabene A, Peng X, Hagemann I S, et al. Tumour hypoxia promotes tolerance and angiogenesis via CCL28 and Treg cells. Nature, 2011, 475: 226-230 


\title{
The progress of tissue resident memory T-cells against tumors
}

\author{
WU QiongLi, KANG ShuangPeng \& WU ChangYou \\ Institute of Immunology, Zhongshan School of Medicine, Sun Yat-sen University, Guangzhou 510080, China
}

Most effector T cells are known to die as only a few of them became memory T cells following immune responses. According to their distribution, function, and transcription factors, memory $\mathrm{T}$ cells can be divided into effector memory $\mathrm{T}$ cells, central memory $\mathrm{T}$ cells, and tissue resident memory $\mathrm{T}$ cells $\left(\mathrm{T}_{\mathrm{RM}}\right)$. The tissue resident memory $\mathrm{T}$ cells do not circulate in the blood and lymph nodes but are found in non-lymphoid tissues, which includes the skin and mucous membranes, urogenital system, brain, respiratory system, lung, and liver tissues. The form of memory $\mathrm{T}$ cells can play the defense response directly and quickly when the pathogens invade the tissues. In addition, the tissue resident memory T cells also play a very important role in the immune responses to tumors, allergic, and autoimmune diseases. The tissue resident memory $\mathrm{T}$ cells include the $\mathrm{CD} 4^{+}$and $\mathrm{CD}^{+} \mathrm{T}$ cells that express cell surface molecules CD69 and/or CD103 and produce cytokines and/or play killing functions during the immune response as well as linking original and adaptive immunity to initiate a systemic immune response. In addition, studies have shown that the function of the tissue resident memory $\mathrm{T}$ cells is controlled by the regulatory $\mathrm{T}$ (Treg) cells in tissues. In this paper, the differentiation of the tissue resident memory $\mathrm{T}$ cells cells and their role in tumors are reviewed.

\section{$\mathbf{T}_{\mathrm{RM}}$, tumor immunity, vaccine}

doi: $10.1360 /$ SSV-2020-0024 